\title{
Ambulatory Assessment Methods to Examine Momentary State-Based Predictors of Opioid Use Behaviors
}

\author{
Albert Burgess-Hull ${ }^{1}$ (D) David H. Epstein ${ }^{1}$ \\ Accepted: 30 November 2020 / Published online: 2 January 2021 \\ (C) This is a U.S. government work and not under copyright protection in the U.S.; foreign copyright protection may apply 2021
}

\begin{abstract}
Purpose of Review Addiction scientists have begun using ambulatory assessment methods_including ecological momentary assessment (EMA), experience sampling, and daily diaries - to collect real-time or near-real-time reports of participants' internal states in their natural environments. The goal of this short review is to synthesize EMA findings from our research group, which has studied several hundred outpatients during treatment for opioid-use disorder (OUD). (We cite pertinent findings from other groups, but have not tried to be comprehensive.) One of our main goals in using EMA is to examine momentary changes in internal states that proximally predict, or concurrently mark, events such as lapses to opioid use.

Recent Findings We summarize findings evaluating several classes of momentary markers or predictors (craving, stress, negative and positive moods, and physical pain/discomfort) of lapses and other states/behaviors. Craving and some negatively valenced mood states are concurrently and prospectively associated with lapses to opioid use during treatment. Craving is also concurrently and prospectively associated with momentary changes in stress and mood. Convincing evidence has not yet emerged for stress as a robust redictor of lapse to opioid use; it appears to be contributory, but neither necessary nor sufficient.

Summary Ambulatory assessment can capture changes in internal states and drug-related behaviors in situ and at high temporal resolution. We recommend research strategies that may increase the clinical and prognostic utility of ambulatory assessment, including denser sampling (i.e., more assessments per day) and more attention to heterogeneity across people and across populations.
\end{abstract}

Keywords Ambulatory assessment · Opioid use disorder · Opioid addiction · Ecological momentary assessment · Momentary predictors $\cdot$ Addiction

\section{Introduction}

Nearly every theory of drug use and addiction recognizes causal roles for both external stimuli and internal states. Traditionally, empirical studies have had to rely on external stimuli administered in laboratory settings and on internal states reported either in laboratory settings or in retrospective/global accounts of daily-life occurrences. Laboratory research offers strong experimental control but has ecological-validity issues that can probably never be fully

This article is part of the Topical Collection on Opioids

Albert Burgess-Hull

albert.burgess-hull@nih.gov

1 Intramural Research Program, National Institute on Drug Abuse, Baltimore, MD, USA surmounted; retrospective reports are subject to considerable biases in recall (e.g., [1-3]). Furthermore, drug-use behaviors in real-world settings are typically discrete, episodic, and difficult to predict. Together, these features make it difficult to fully characterize the dynamics of drug use in real-world contexts.

To circumvent limitations of laboratory experiments and retrospective reports, addiction scientists have begun using ambulatory assessment methods to collect real-time reports of a patient's internal states in their natural environment. These methods - which include ecological momentary assessment [4], the experience-sampling method (ESM; [5]), and daily diary methods - have provided critical insights into the causes and consequences of substance use in real-world settings, and have the potential to open up new avenues for treatment. (A note on terminology: there is no uniform distinction between ESM and EMA, but ESM often refers to collection of data at random or regular times not determined 
by the respondent/participant, while EMA often refers to a combination of ESM, via "prompted" entries, with participant-initiated data collection, via "event-contingent entries" that mark moments of interest such as drug use.)

The goal of this short review is to synthesize our recent findings using EMA to examine momentary changes that proximally predict, or concurrently mark, instances of drugrelated behaviors in people who use opioids nonmedically and/or have opioid use disorder (OUD). For the purpose of this review, we will focus on internal states rather than environmental contexts. We start by briefly describing how ambulatory assessment methods have been used in OUD research. We then summarize our major published findings. (We cite relevant findings from other groups as well but have not tried to provide complete coverage.) We conclude with a short synthesis of the findings and their implications.

\section{Ambulatory Assessment in Studies of Opioid Use or OUD}

As far as we can determine, the first mobile-device-based study of what is now called OUD was conducted by our research group in 2005-2006 [6•] (See Table 1 for a summary of our major EMA studies). When we started that study, EMA (with PalmPilots) had been successfully deployed with large samples of cigarette smokers [3], but had rarely been deployed to study drugs that are acutely intoxicating. Intoxication seemed likely to preclude provision of EMA reports, and a small feasibility study in people with alcohol use disorder (AUD) had suggested that that was often the case [7]. However, subsequent feasibility studies with users of MDMA [8] and crack cocaine [9] had been more promising, at least in terms of adherence. To study OUD with EMA, we enrolled people to whom we were providing free daily methadone treatment (along with weekly counseling and case management), many of whom continued to use some heroin (and cocaine), despite individualized dosing of methadone. We were able to use free treatment as an incentive for adherence to study requirements - an agreement we carefully explained to our study participants from the first contact onwards, so as to avoid undue influence or coercion. Our consent forms explained the spirit in which we were collecting the EMA data:

The main purpose of this study is to learn why people may keep using cocaine and heroin when they're trying not to. Some researchers have studied this by talking to people who have already started using again. But people see things differently in hindsight. We may learn more if we see how people's lives are going before they start using again. We also want to study the exact moment that people use-or the moment when they resist the temptation. We can do this by giving people electronic diaries (EDs) to record where they are and what they're doing several times a day. EDs have been used in studies of cigarette smokers who were trying to quit. This study will be one of the first to use EDs in cocaine and heroin users. You qualify for the study because you use cocaine and heroin and are seeking treatment.

When we met with participants to review the consent form, we emphasized that EMA was an opportunity to make their voices heard, correcting longstanding assumptions about OUD that investigators like us had made on the basis of limited data. This seemed to resonate with participants, and the rates of adherence (and apparent quality of the data) were accordingly good $\left[6^{\bullet}\right]$.

As our consent-form language indicates, we collected a combination of the following:

- Randomly prompted entries. These were assessments of activity, context, and mood at the moments of randomly timed prompts, with the restriction that prompts occurred only during the participant's self-reported typical waking hours for each day of the week. One major purpose of random-prompt entries is to estimate the base rates of events (e.g., being alone or watching TV) and the background intensities of rated states (e.g., boredom or positive mood). The resultant data can be of inherent interest and can also flag illusory correlations in event-driven entries (e.g., seeming associations between a behavior and a context or mood, reflecting confirmation bias on the part of participants or investigators).

- Event-contingent entries. These were made at the participant's discretion, always including heroin or cocaine use, and, depending on the study, also including discrete increases of either drug craving or stress. Unlike randomprompt entries, they can theoretically capture every instance of an event of interest, regardless of how rare it is or whether it occurs outside random-prompt hours. In practice, even reports of externally verifiable events (such as drug use in participants who have agreed to thriceweekly urine testing) cannot be checked for completeness or temporal precision. This could be considered a limitation of EMA, but it is also the reason EMA exists. Eventcontingent entries cannot currently be obviated by continuous passive monitoring with biosensors or smartphone sensors.

In our later studies, we also collected the following:

- End-of day entries. These were prompted each day approximately $1 \mathrm{~h}$ before the participant's self-reported bed/sleeping time. They are useful for global assessments 


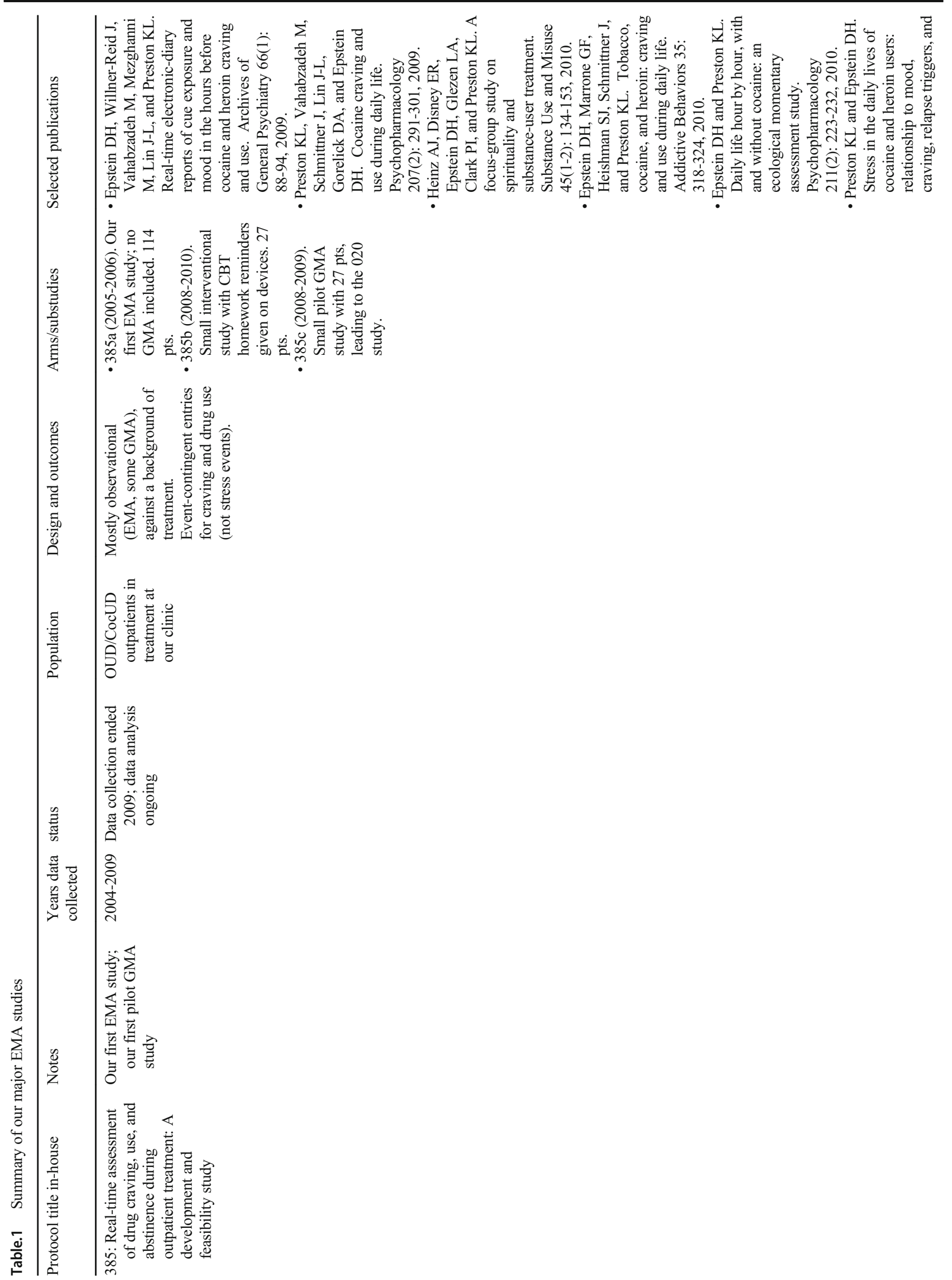




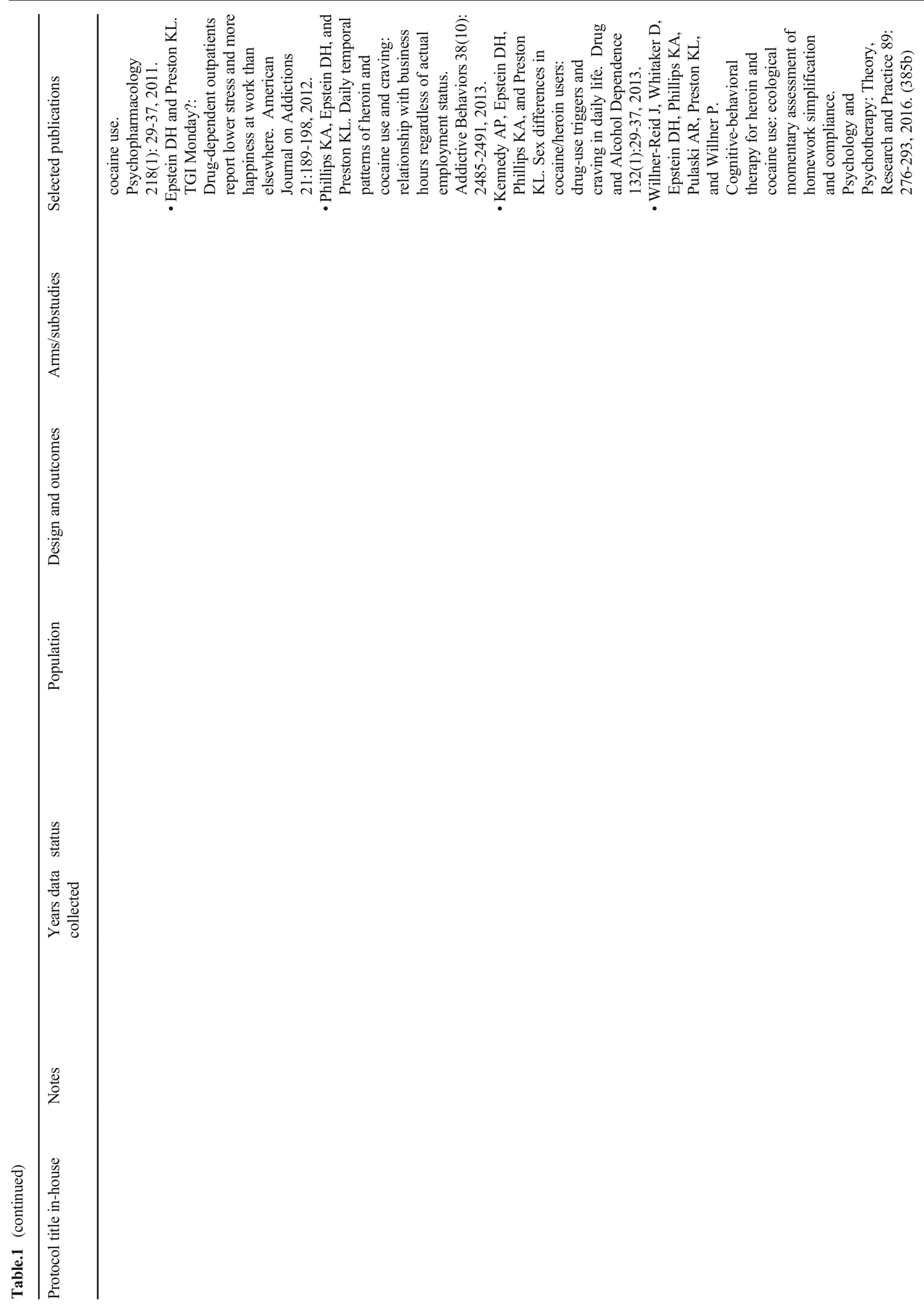




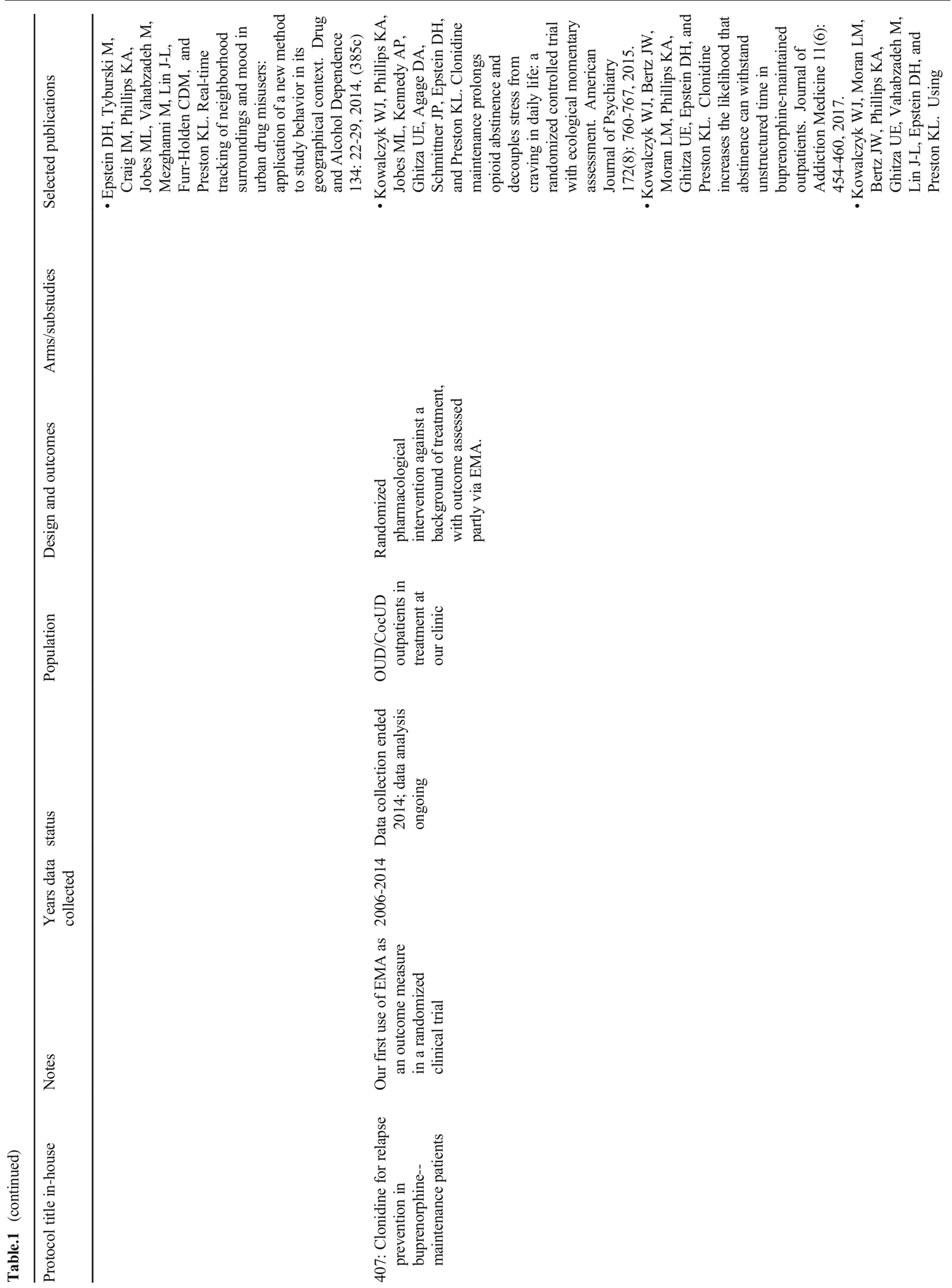




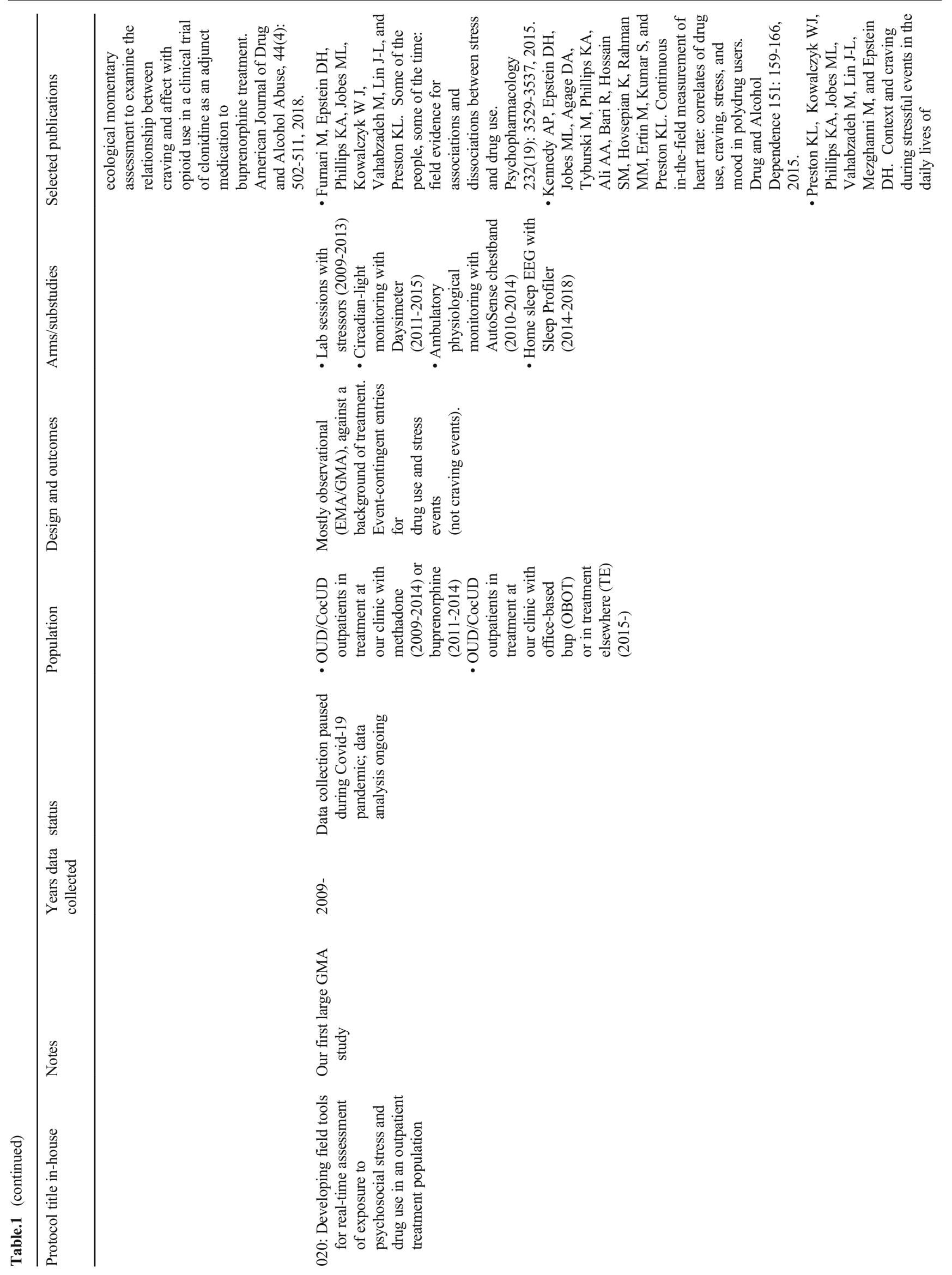




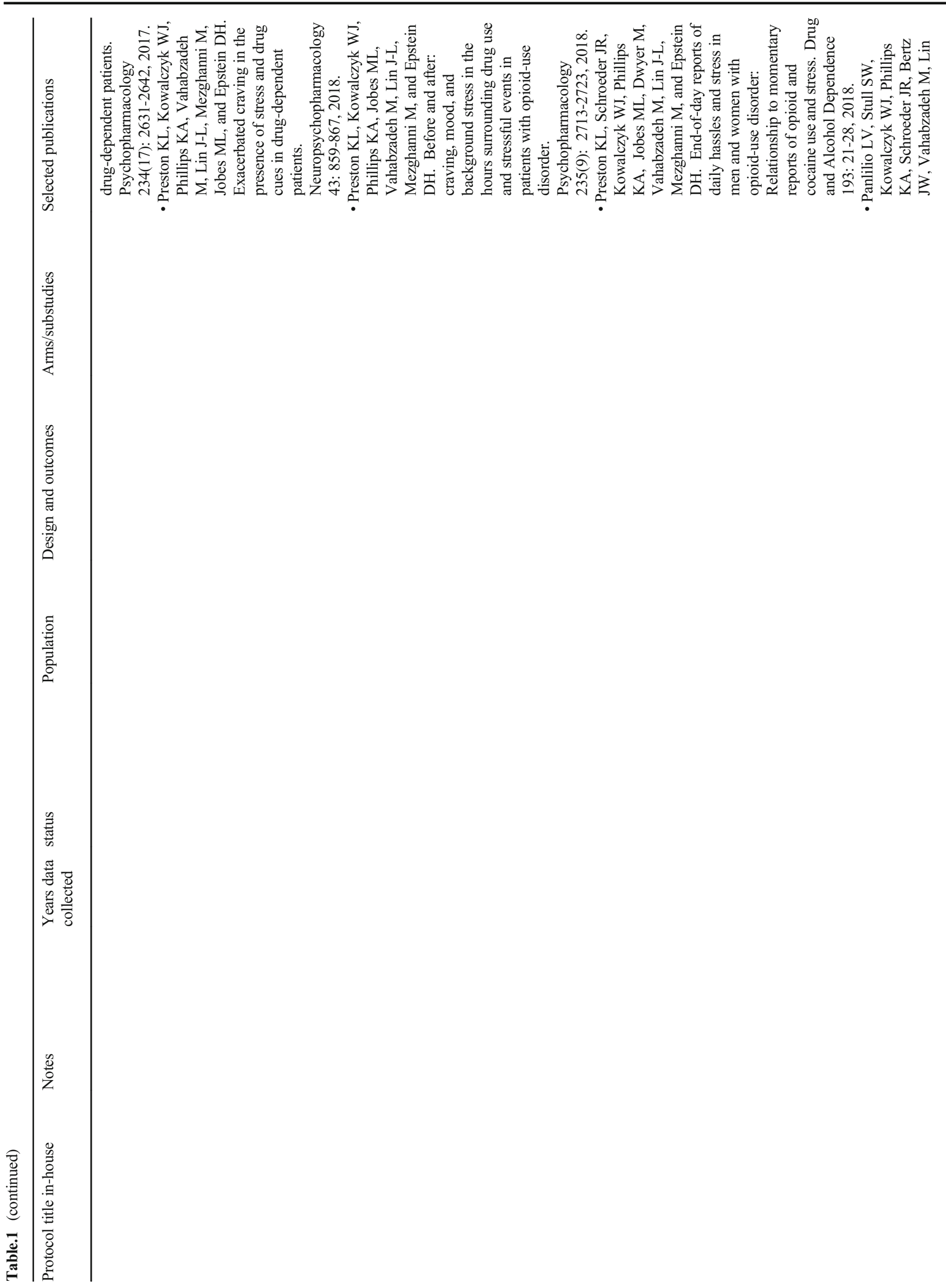




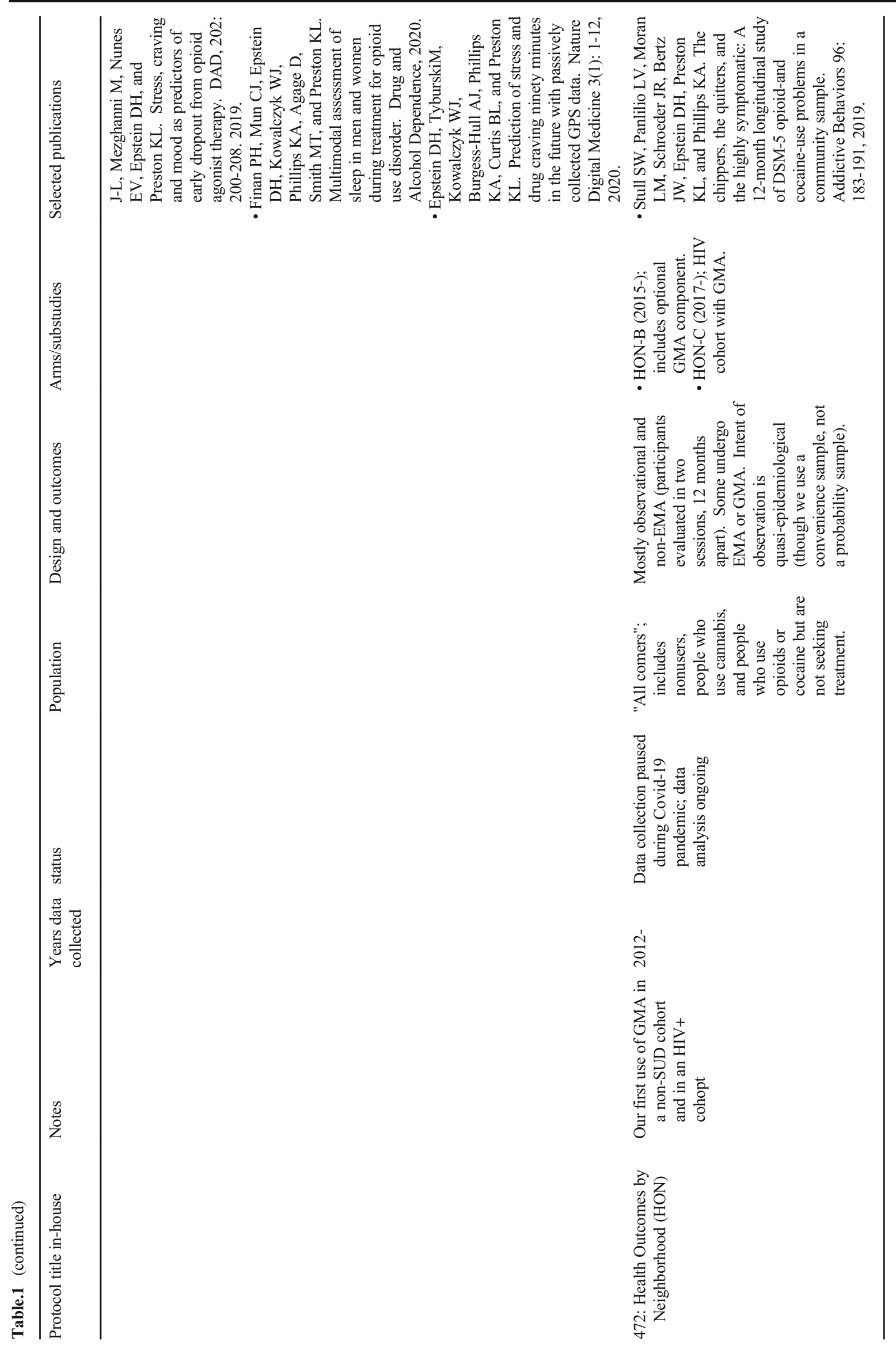


of a whole day's events and for reports of events that might have been unreported in real time.

One of our first objectives was to test whether instances of drug use or craving were preceded by increases in randomprompt reports of putative triggers. A list of such triggers, identified using retrospective interviews, had been developed into a much-cited taxonomy $[10,11]$ and subsequently operationalized into a questionnaire [12•], from which we adapted many of our random-prompt assessments.

In the next sections, we summarize our findings on craving as a dynamic marker or predictor of opioid use and other momentary states or behaviors. We then summarize our findings on other internal states that may be dynamic markers or predictors of opioid use (and other behaviors) and of craving; most of these markers or predictors correspond to elements of the Marlatt \& Gordon taxonomy, such as stress, negative moods, positive moods, and physical discomfort.

\section{Momentary Self-Reported State-Based Markers/Predictors of Opioid-Use Behaviors and Other Momentary States/Behaviors}

Discrete episodes of opioid use, like most other behaviors, result from a complex interplay between environmental stimuli and internal processing of those stimuli. However, it is safe to say that the proximal mediators of all behavioral outputs (except reflexes) are internal states. To the extent that changes in internal states are accessible to consciousness, one of the best ways to capture the dynamics of those changes is through ambulatory assessment. The next sections briefly summarize some of the extant literature in the context of nonmedical opiate use or OUD.

\section{Craving}

Drug craving - commonly defined as a conscious, reportable urge to use drugs - is one of the most frequently researched constructs in the opioid use literature. Despite craving's central role in many contemporary theories of addiction and relapse (e.g., [13-15]), there is still debate about the extent to which craving is causally associated with use. Ambulatory assessment alone cannot resolve the debate, but can ground it more firmly in data that show how changes in craving accompany instances of use, including the circumstances under which the two are dissociable.

\section{Craving and Drug-Use Outcomes}

Ambulatory assessment studies of drug craving in people with OUD have found increases in craving in the days, hours, or minutes preceding use. However, the robustness of these associations appears to depend on the substance specificity of the craving (e.g., opioid vs. psychostimulant craving, as many study participants use drugs from both classes) and the time elapsed between the assessment of craving and use.

We found that higher levels of opioid craving predicted next-day opioid use [16, 17]; others have found that opioid craving intensity predicted opioid use in the next $4 \mathrm{~h}[18]$ and that higher levels of opioid craving were associated with concurrent ratings of opioid use [18]. We have shown that levels of heroin craving (and cocaine craving) were higher on the days when patients reported cocaine use than on days of verified cocaine abstinence [19].

During a 7-day inpatient treatment study, Marhe et al. [20] found that between-person differences in heroin (or cocaine) craving were associated with earlier relapse after discharge; however, within-person increases in craving did not predict the timing of relapses. Finally, a randomized trial in our clinic showed that clonidine augmentation of buprenorphine maintenance protected against stress-induced lapses to opioid use - but when use did occur during clonidine augmentation, it was predicted by especially strong increases in craving the previous day [17]. This finding illustrates how ambulatory assessment can be integrated into randomized trials to offer clues as to how and when an experimental treatment works (or does not).

In short, ambulatory assessment studies have found a robust link between craving and subsequent drug use, with the strongest evidence observed for opioids. Although momentary increases in craving can predict drug use on multiple timescales, the strength of the relationship appears to increase as the lag decreases. However, the decay of this relationship is still not well understood, and we are only aware of one study [18] that has directly examined associations between craving and drug use at different timescales within the same sample. Future research should further clarify how the strength of association between craving and drug use changes at different timescales within the same or similar samples.

\section{Craving and Non-drug Use Outcomes}

In medication-assisted treatment for OUD, we found that both opioid and cocaine craving increase in the hours leading up to participant-initiated reports of discrete increases in stress [21 • 22•, 23•]. Serre et al. [18] found that higher craving intensity was associated with higher concurrent ratings of sad or anxious moods, along with higher negatively valenced ratings of a recent event.

\section{Stress}

In this review, we discuss stress separately from the overlapping concept of "negative affect," partly because stress has been singled out as a causal factor in most theories of the 
development and persistence of OUD and other SUDs (including theories that are better known for emphasizing craving, e.g., [24]). Where negative affect is usually defined to include both high-arousal and low-arousal states (such as sadness), stress is usually defined as a high-arousal state, and its emotional valence is not exclusively negative [25]. In ambulatory assessment of OUD, stress has been studied both as a fluctuating "background" state (via randomly prompted entries) and as a discrete event (via participant-initiated entries).

\section{Stress and Drug-Use Outcomes}

Most studies of stress as a momentary predictor of opioid use have focused on patients in medication-assisted treatment. Results have tended to be less conclusive than those for craving as a momentary predictor.

In two studies from our group, higher levels of stress in random-prompt entries predicted greater likelihood of cocaine or opioid use in the next 2 days [16, 17]. During days of cocaine (but not opioid) use, stress levels appeared to continually increase over the course of the day [19]. On a time scale of $5 \mathrm{~h}$ before instances of opioid use (during which other predictors of use show robust linear increases, as discussed in a later section), we have never found a linear increase in random-prompt ratings of stress $[19,21 \bullet, 22 \bullet, 23 \bullet, 26]$. At most, we have seen more complex associations, such as higher stress $2 \mathrm{~h}$ before use, with a decrease $1 \mathrm{~h}$ before use [21 $, 22 \bullet, 23 \bullet]$. We cannot tell whether this reflects a true nonlinear change over time, because we gave participants no more than five random prompts per day (and often fewer), so our inferences about temporal trends were based on data aggregated across participants in multilevel models. There were almost no instances in which one participant contributed stress data at two closely spaced time points (e.g., hours -2 and -1 ) before a specific instance of drug use. We discuss that issue toward the end of this paper.

We have also assessed stress as a discrete event, telling participants to make an EMA entry "whenever you feel more stressed, anxious, or overwhelmed than usual" (and asking, as part of the entry, which adjectives apply). We looked for associations between stress-event entries and subsequent drug use, using a time scale of days rather than hours, because the data were less dense than randomprompt data [27•]. We did not find an increase in the number of stress reports in the 3 three days leading up to opioid (or cocaine) use. However, the self-reported severity of the stressor increased in the 3 days before cocaine use, but not heroin use. In a separate sample of participants (from the randomized trial of clonidine maintenance cited above), we found that higher levels of random-prompt stress predicted opioid use on a time scale of 2 days [17].

\section{Stress and Non-drug Use Outcomes}

In patients with OUD, we found that increases in randomprompt ratings of stress were associated with concurrent increase in craving for opioids (along with craving for cocaine and tobacco) [21•, 22•, 23•, 26, 28•, 29]. Furthermore, participant-initiated reports of stress events were rated higher in severity when accompanied by increases in opioid (or cocaine) craving [29, 30]. However, we did not find a linear increase in random-prompt ratings of stress in the $5 \mathrm{~h}$ leading up discrete episodes of opioid craving [26].

We have found that momentary ratings of stress were associated with concurrent self-reported negative-affect states (e.g., tired, bored, irritated, frustrated, worried, uncomfortable, sad) and inversely associated with positiveaffect states (e.g., happy, relaxed). Higher stress ratings were also associated with increases in thoughts about drugs (e.g., "wanting to see what would happen if I tried cocaine/ heroin," "was tempted out of the blue to use") [26]. Background stress levels appeared to change in a complex manner in the $5 \mathrm{~h}$ preceding a stress event, first decreasing, and then increasing $[21 \bullet, 22 \bullet, 23 \bullet]$; again, we cannot be sure that this reflects a true within-person pattern. Finally, more frequent reports of stress events were associated with endof-day reports of feeling angry, annoyed, or afraid, and inversely associated with end-of-day reports of feeling content $[21 \bullet, 22 \bullet, 23 \bullet]$.

We have found preliminary evidence that the association between stress states and craving at the momentary level is moderated by sex and by medication status. For example, momentary stress was more strongly associated with concurrent ratings of opioid craving in women than in men (while the association between stress and cocaine craving was greater for men than women) [29]. In our clonidine trial, increases in stress were associated with higher concurrent levels of heroin craving, and this relationship was attenuated for patients randomized to clonidine maintenance [28•].

\section{Positive and Negative Affect}

A note on terminology: several different distinctions, not entirely compatible with each other, have been made between affect and mood. In this review, we use them interchangeably to refer to valenced states that, in EMA studies, are usually assessed by participant ratings of adjectives (happy, sad, angry, afraid, etc.) at study-specific timescales such as "right now."

Positive affect (PA) and negative affect (NA), whether assessed as composite constructs or as individually labeled moods, can each influence opioid-related behaviors $[31,32 \bullet]$. 


\section{Affect and Drug-Use Outcomes}

We have found that higher ratings of boredom, irritation, and tiredness predicted opioid (and cocaine) use during the next 2 days $[16,17]$ - as did lower ratings of relaxation or happiness $[16,17]$. We also found that negative mood statesincluding feeling bored, worried, sad, criticized, annoyed, hopeless, discouraged, uneasy, uncomfortable, fatigued, or worn out - increased in the hours before cocaine or opioid use $[6 \bullet, 21 \bullet, 22 \cdot, 23 \bullet]$. Reports of having been "in a good mood...in the past hour" increased in the hours preceding cocaine use, but not opioid use [6•].

We have found some evidence that the proximal effect of PA and NA on drug use can be moderated by sex, medication type, and, unexpectedly, by hepatitis $\mathrm{C}$ status. Men were more likely than women to attribute their heroin use (or cocaine use) to feeling uncomfortable [33•]. In our randomized trial of clonidine augmentation, opioid use in the next 2 days was predicted by higher levels of feeling tired, bored, and irritated, and by lower levels of happiness [17]. Finally, we found that patients with hepatitis $\mathrm{C}$ (largely asymptomatic in our sample) were more likely than others to attribute their heroin use (and cocaine use) to several of the triggers from Marlatt's taxonomy, including feeling bored, uncomfortable, worried, sad, feeling like others were being critical of them, or being in a good mood [34]; we do not know the mechanism of this difference.

Two studies by other investigators have examined changes in NA and PA and drug use among patients being treated for chronic pain. One study found that NA (measured by a composite of anxiety and depression questionnaires) was associated with misuse of prescription opioids [35•]. Another study, which examined the effect of a mindfulness-based intervention to reduce pain and opioid misuse, found that improvements in PA were associated with a reduction in the likelihood of misusing opioids [36].

\section{Affect and Non-drug Use Outcomes}

Higher momentary levels of NA are associated with higher levels of craving and sometimes stress. For example, we found that higher levels of NA in the past hour were concurrently linked with higher momentary cocaine craving and stress, and an increase in participant-initiated reports of stress events [21•, $22 \bullet, 23 \bullet, 33 \bullet$. NA states, such as feeling worried, criticized by others, bored, or angry, increased linearly in the $5 \mathrm{~h}$ preceding a report of a heroin-craving event $\left[6^{\bullet}\right]$. In our randomized study of clonidine augmentation, higher momentary ratings of feeling tired, bored, or irritated were associated with higher concurrent ratings of heroin craving [28•].

Two studies by other investigators - one conducted among patients in residential treatment for OUD and the other among patients in treatment for nonmedical use of prescription drugs - found that opioid craving levels increased on days when NA was higher than usual $[37,38]$.

In one other study by other investigators, higher negatively valenced ratings of a recent event were predictive of lower ratings of opiate craving approximately $4 \mathrm{~h}$ later [18]. This was the only study we found that reported an inverse association between NA and craving.

Our group and others have also found that momentary ratings of PA can predict higher craving, but this seems to apply mostly to cocaine craving, not opioid craving $[6 \bullet, 33 \bullet]$. In our randomized clonidine study, higher ratings of momentary PA were associated with decreases in concurrent opioid craving [28•]. Other investigators have also found that opioid craving was lower on days when PA is higher than usual [38], and this relationship appears to be especially strong for patients with low overall levels of PA during treatment [37]. Finally, we have found some evidence that sex can moderate the effect of PA and NA on stress and craving. Past-hour NA is more strongly associated with momentary ratings of stress and cocaine craving in women than in men, and some past-hour PA states (such as "good mood") are more strongly associated with cocaine craving in women than in men [33॰].

A few studies by other investigators have evaluated PA and NA in relation to opioid-related outcomes for patients with chronic pain. Two studies found that higher levels of baseline or momentary NA were associated with heightened levels of opioid craving $[35 \cdot 39]$. Another found that increases in momentary PA were associated with decreased concurrent levels of opioid craving [40].

\section{Pain}

Iatrogenic addiction to opioids is a low-probability event in relative terms, but is nonetheless prevalent because opioids have been so widely prescribed for pain [41-43]. Furthermore, a large proportion of patients in methadone maintenance treatment for OUD report chronic pain conditions (e.g., $[44,45])$. Nonetheless, our group's work with ambulatory assessment in this area has been limited, so we focus here on work from other investigators.

Day-to-day elevations in pain are concurrently associated with increases in ratings of opioid craving - but not very strongly. For example, in a 14-day study of chronic-pain patients, Martel et al. [39] found that within-person changes in pain intensity explained less than $5 \%$ of the variance in patients' momentary ratings of craving. On the other hand, in a daily-diary study of chronic-pain patients conducted over 1 day, Martel et al. [35•] found no relationship between pain intensity and opioid craving in the past $24 \mathrm{~h}$. However, Martel et al. [35•] did find that pain intensity was associated with prescription opioid misuse (measured by the Current Opioid Misuse Measure; [46]) and NA (in the past $24 \mathrm{~h}$ ). 


\section{Conclusion and Future Directions}

Findings from ambulatory-assessment studies underscore the value of capturing changes in internal states and drug-related behaviors in situ and at high temporal resolution, as a probe for subtle, unexpected associations, and as a test for longpresumed associations.

We have consistently observed that craving and some negatively valenced mood states (negative affect) are concurrently and prospectively associated with instances of drug use in patients with OUD. In turn, craving (for multiple drug classes) is concurrently and prospectively associated with momentary changes in stress and mood (with the direction of the association depending on the valence of the reported mood). Positive mood states also appear to be associated with cocaine craving and use in OUD; however, more research is needed to clarify these associations.

Perhaps surprisingly, convincing evidence has not yet emerged for stress as a robust predictor of lapse to opioid use. Although we have found increased stressor severity in the days (not hours) preceding opioid (and cocaine) use, the heterogeneity in this association is as prominent as the association itself. We believe that clearer conclusions about stress and lapse likely depend on moderators at both the within-person level (e.g., momentary appraisals of stress) and the between-person level (e.g., trait-like levels of chronic stress or reactivity to stress), and also on a clearer characterization of the dynamic relationship between momentary stress and the mediators of its relationship to lapse. One such mediator could be craving; there is convincing laboratory evidence that stressors increase craving in patients with OUD (e.g., [47, 48]). Furthermore, our group and others have found that increases in momentary stress are associated with concurrent increases in craving for opioids (e.g., [28•, 29]). However, to our knowledge, ambulatory assessment studies have yet to directly examine the temporal ordering of these associations around lapse events. Preliminary analyses from our group also suggest that the lapserelevant effects of momentary changes in stress levels during treatment may depend on longer-term between-person differences in stress levels. We recommend that future ambulatory research further clarify these interdependencies.

We agree with other authors (e.g., [49•]) that the prognostic value of stress, craving, and other momentary state-based predictors will only be realized after clearer characterization of the factors that moderate the relationship between these constructs and drug use. Consequently, we encourage researchers interested in using ambulatory assessment for OUD research to focus on testing potential moderators drawn from both the extant ambulatoryassessment work and laboratory research (e.g., stressor predictability; see e.g., $[50,51])$. Translating laboratory research to more ecologically valid contexts is vital for refining theories of addiction and disease maintenance and can inform targets for just-in-time adaptive interventions. Taken together, results from ambulatory assessment studies in patients with OUD suggest that the relationships between internal states and behavioral outcomes are rarely independent, and are instead bidirectional and multiplicative (see $[21 \bullet, 22 \bullet, 23 \bullet])$. Measures of dynamic processes such as instability, temporal dependence, or oscillation (e.g., [52•, 53]) may help further clarify these dynamic relationships.

For within-person processes, care should be taken when drawing conclusions about dynamic associations at timeframes narrower than whole days. Our group-like others - has typically collected random-prompt reports from participants no more than five times per day. This means that when we examine associations on time scales of a few hours, we do so by aggregating data both across and within participants. Thus, associations are composed of heterogeneous patient-types and heterogeneous contexts (e.g., weekday vs. weekend responses). Multilevel modeling can go only so far in disaggregating within-person from between-person effects. To assess the true functional form of the relationships of interest (e.g., whether drug use is best predicted by a nonlinear change in stress severity over hours, perhaps U-shaped or even more complex), we suggest the use of denser sampling schemes [54] and shorter survey's with more targeted questions (see e.g., [55]). Measurement tools that collect data types that require little to no participant burden are likely to be the most efficient way to collect dense data at a more fine-grained temporal resolution. Some examples of useful approaches that have been implemented by our group and others include GPS tracking [56], smartphone accelerometer data to sense intoxication [57], and mobile phone keystroke metadata (e.g., [58]).

No matter how dense the sampling and no matter how finegrained the data, no theory is likely to comport with observed relationships among mood/stress, craving, and lapse across people and situations if the theory is framed universally or inflexibly. As noted by Davis-Stober and Regenwetter [59], theories of behavior will be most robust when they explicitly incorporate the inescapable observation that not everyone responds identically to identical circumstances. This appears to be as true for drug-related behaviors in people with SUDs as it is for other behaviors in essentially any population. EMA can elucidate this heterogeneity, but only if the data are collected and analyzed with heterogeneity in mind.

\section{References}

Papers of particular interest, published recently, have been highlighted as:

- Of importance

1. Collopy F. Biases in retrospective self-reports of time use: an empirical study of computer users. Manag Sci. 1996;42(5):758-67. https://doi.org/10.1287/mnsc.42.5.758. 
2. Miron-Shatz T, Stone A, Kahneman D. Memories of yesterday's emotions: does the valence of experience affect the memoryexperience gap? Emotion. 2009;9(6):885-91.

3. Shiffman, S., Hufford, M., Hickcox, M., Paty, J. A., Gnys, M., \& Kassel, J. D. (1997). Remember that? A comparison of real-time versus retrospective recall of smoking lapses. In Journal of Consulting and Clinical Psychology (Vol. 65, Issue 2, pp. 292300). American Psychological Association. https://doi.org/10. 1037/0022-006X.65.2.292.a.

4. Stone AA, Shiffman S. Ecological momentary assessment (EMA) in behavorial medicine. Annals of Behavioral Medicine. 1994.

5. Larson, R., \& Csikszentmihalyi, M. (2014). The experience sampling method. In Flow and the foundations of positive psychology (pp. 21-34). Springer.

6. Epstein DH, Willner-Reid J, Vahabzadeh M, Mezghanni M, Lin JL, Preston KL. Real-time electronic diary reports of cue exposure and mood in the hours before cocaine and heroin craving and use. Arch Gen Psychiatry. 2009;66(1):88-94. https://doi.org/10.1001/ archgenpsychiatry.2008.509 This was the first published set of analyses from our group's first study using EMA in OUD. We examined whether items from the Marlatt taxonomy of triggers, assessed in random-prompt entries, increased in frequency during the 5 hours preceding entries for craving for or use of drug (heroin or cocaine).

7. Litt MD, Cooney NL, Morse P. Ecological momentary assessment (EMA) with treated alcoholics: Methodological problems and potential solutions. In Health Psychology. American Psychological Association. 1998;17(1):48-52. https://doi.org/10.1037/02786133.17.1.48

8. Lukas SE, Stull M, Cowan RL. Polydrug abuse patterns with MDMA (Ecstasy) among young adults. Drug Alcohol Depend. 2002;66(suppl 1):S107.

9. Freedman M, Pulley L, Abdolrussa B, McNamara C, Milby J, and Schumacher J. Ecological momentary assessment of crack cocaine relapse risk. In: 64th annual scientific meeting of the College on Problems of Drug Dependence, San Juan, Puerto Rico, 2002.

10. Cummings C, Gordon JR, Marlatt GA. Relapse: prevention and prediction. The Addictive Behaviors. 1980:291-321.

11. Marlatt GA, Gordan J. Relapse prevention: Maintenance strategies in the treatment of addictive behaviors. Guilford press. 1985.

12. Heather N, Stallard A, Tebbutt J. Importance of substance cues in relapse among heroin users: comparison of two methods of investigation. Addict Behav. 1991;16(1-2):41-9.

13. Baker TB, Piper ME, McCarthy DE, Majeskie MR, Fiore MC. Addiction motivation reformulated: an affective processing model of negative reinforcement. Psychol Rev. 2004;111(1):33.

14. Wise RA, Koob GF. The development and maintenance of drug addiction. Neuropsychopharmacology. 2014;39(2):254-262.

15. Khantzian EJ. The self-medication hypothesis of substance use disorders: a reconsideration and recent applications. Harvard Review of Psychiatry. 1997;4(5):231-44.

16. Epstein DH, Preston KL. TGI Monday?: drug-dependent outpatients report lower stress and more happiness at work than elsewhere. Am J Addict. 2012;21(3):189-98.

17. Kowalczyk WJ, Moran LM, Bertz JW, Phillips KA, Ghitza UE, Vahabzadeh M, et al. Using ecological momentary assessment to examine the relationship between craving and affect with opioid use in a clinical trial of clonidine as an adjunct medication to buprenorphine treatment. The American Journal of Drug and Alcohol Abuse. 2018;44(5):502-11.

18. Serre F, Fatseas M, Denis C, Swendsen J, Auriacombe M. Predictors of craving and substance use among patients with alcohol, tobacco, cannabis or opiate addictions: commonalities and specificities across substances. Addict Behav. 2018;83:123-9.
19. Preston KL, Vahabzadeh M, Schmittner J, Lin J-L, Gorelick DA, Epstein DH. Cocaine craving and use during daily life. Psychopharmacology. 2009;207(2):291-301.

20. Marhe R, Waters AJ, van de Wetering BJM, Franken IHA. Implicit and explicit drug-related cognitions during detoxification treatment are associated with drug relapse: an ecological momentary assessment study. J Consult Clin Psychol. 2013;81(1):1-12.

21. Preston KL, Kowalczyk WJ, Phillips KA, Jobes ML, Vahabzadeh $\mathrm{M}$, Lin JL, et al. Before and after: craving, mood, and background stress in the hours surrounding drug use and stressful events in patients with opioid-use disorder. Psychopharmacology. 2018a;235(9):2713-23. https://doi.org/10.1007/s00213-018-49669 This was the first publication in which we examined the hours following episodes of drug use (opioid or cocaine) rather than just the hours preceding them. A more direct approach, which we have incorporated into our ongoing work, is to issue systematic follow-up prompts in the minutes or hours after an event-contingent entry.

22. Preston KL, Kowalczyk WJ, Phillips KA, Jobes ML, Vahabzadeh $\mathrm{M}$, Lin J-L, et al. Exacerbated craving in the presence of stress and drug cues in drug-dependent patients. Neuropsychopharmacology. 2018b;43(4):859-67 In these analyses, we used EMA to help resolve a puzzling discrepancy in human laboratory studies, where stress and drug cues, as craving triggers, often seemed to compete with each other rather than exacerbate each other's effects. As in our "some of the people, some of the time" paper (Furnari et al., 2015), there was a modal finding (of additive exacerbation), but its strength varied (for some participants, the exacerbation was synergistic), as did its direction (for a few participants, there was competition rather than exacerbation).

23. Preston KL, Schroeder JR, Kowalczyk WJ, Phillips KA, Jobes ML, Dwyer M, et al. End-of-day reports of daily hassles and stress in men and women with opioid-use disorder: relationship to momentary reports of opioid and cocaine use and stress. Drug Alcohol Depend. 2018c;193:21-8.

24. Robinson TE, Berridge KC. The neural basis of drug craving: An incentivesensitization theory of addiction. In Brain Research Reviews. Elsevier Science. 1993;18(3):247-291.

25. Folkman $\mathrm{S}$. The case for positive emotions in the stress process Anxiety Stress Coping. 2008;21(1):3-14.

26. Preston KL, Epstein DH. Stress in the daily lives of cocaine and heroin users: relationship to mood, craving, relapse triggers, and cocaine use. Psychopharmacology. 2011;218(1):29-37.

27. Furnari M, Epstein D, Phillips K, Jobes M, Kowalczyk W, Vahabzadeh M, et al. Some of the people, some of the time: field evidence for associations and dissociations between stress and drug use. Psychopharmacology. 2015;232. https://doi.org/10.1007/ s00213-015-3998-7 This was our group's first published analysis on EMA reports of stress as a discrete event rather than a quantitatively rated background state. We found that stress, assessed this way, was prospectively associated with drug use (a previously elusive association), but, as the paper title indicates, exceptions were common and are probably important.

28. Kowalczyk WJ, Phillips KA, Jobes ML, Kennedy AP, Ghitza UE, Agage DA, et al. Clonidine maintenance prolongs opioid abstinence and decouples stress from craving in daily life: a randomized controlled trial with ecological momentary assessment. Am J Psychiatr. 2015;172(8):760-7 This was a randomized clinical trial in which EMA was integrated into our outcome measures. By using EMA in that fashion, we were able to show not only that our experimental treatment was effective (it lengthened the time to lapse to opioid use), but also that it worked via the behavioral mechanism we had hypothesized (decoupling of 
stress from craving) from the laboratory-animal research we were translating.

29. Moran LM, Kowalczyk WJ, Phillips KA, Vahabzadeh M, Lin J-L, Mezghanni M, et al. Sex differences in daily life stress and craving in opioid-dependent patients. The American Journal of Drug and Alcohol Abuse. 2018;44(5):512-23.

30. Preston KL, Kowalczyk WJ, Phillips KA, Jobes ML, Vahabzadeh $\mathrm{M}$, Lin J-L, et al. Context and craving during stressful events in the daily lives of drug-dependent patients. Psychopharmacology. 2017;234(17):2631-42.

31. Cheetham A, Allen NB, Yücel M, Lubman DI. The role of affective dysregulation in drug addiction. Clin Psychol Rev. 2010;30(6): 621-34.

32. Finan PH, Remeniuk B, Dunn KE. The risk for problematic opioid use in chronic pain: what can we learn from studies of pain and reward? Prog Neuro-Psychopharmacol Biol Psychiatry. 2018;87: 255-62. https://doi.org/10.1016/j.pnpbp.2017.07.029.

33. Kennedy AP, Epstein DH, Phillips KA, Preston KL. Sex differences in cocaine/heroin users: drug-use triggers and craving in daily life. Drug and Alcohol Dependence. 2013;132(1-2):29-37 This is one of two publications in which we examined sex differences in OUD patients undergoing EMA. We found that men and women in our sample were more alike than different in their opioidrelated behaviors, but that the Marlatt taxonomy of triggers applied more to men's lapses and craving than to women's, underscoring the need for more inclusion of women in formative research.

34. Phillips KA, Epstein DH, Vahabzadeh M, Mezghanni M, Lin J-L, Preston KL. Substance use and hepatitis C: an ecological momentary assessment study. Health Psychol. 2014;33(7):710-9.

35. Martel MO, Dolman AJ, Edwards RR, Jamison RN, Wasan AD. The association between negative affect and prescription opioid misuse in patients with chronic pain: the mediating role of opioid craving. J Pain. 2014;15(1):90-100.

36. Garland EL, Bryan CJ, Finan PH, Thomas EA, Priddy SE, Riquino MR, et al. Pain, hedonic regulation, and opioid misuse: modulation of momentary experience by mindfulness-oriented recovery enhancement in opioid-treated chronic pain patients. Drug Alcohol Depend. 2017;173:S65-72.

37. Huhn AS, Harris J, Cleveland HH, Lydon DM, Stankoski D, Cleveland MJ, et al. Ecological momentary assessment of affect and craving in patients in treatment for prescription opioid dependence. Brain Res Bull. 2016;123:94-101.

38. Lydon-Staley DM, Cleveland HH, Huhn AS, Cleveland MJ, Harris J, Stankoski D, et al. Daily sleep quality affects drug craving, partially through indirect associations with positive affect, in patients in treatment for nonmedical use of prescription drugs. Addict Behav. 2017;65:275-82.

39. Martel MO, Finan PH, McHugh RK, Issa M, Edwards RR, Jamison $\mathrm{RN}$, et al. Day-to-day pain symptoms are only weakly associated with opioid craving among patients with chronic pain prescribed opioid therapy. Drug Alcohol Depend. 2016;162:130-6.

40. Garland EL, Hanley AW, Kline A, Cooperman NA. Mindfulnessoriented recovery enhancement reduces opioid craving among individuals with opioid use disorder and chronic pain in medication assisted treatment: ecological momentary assessments from a stage 1 randomized controlled trial. Drug Alcohol Depend. 2019;203:615 .

41. Epstein DH, Heilig M, Shaham Y. Science-based actions can help address the opioid crisis. Trends Pharmacol Sci. 2018;39(11):9116. https://doi.org/10.1016/j.tips.2018.06.002.

42. Fishbain DA, Cole B, Lewis J, Rosomoff HL, Rosomoff RS. What percentage of chronic nonmalignant pain patients exposed to chronic opioid analgesic therapy develop abuse/addiction and/or aberrant drug-related behaviors? A structured evidence-based review. Pain Med. 2008;9(4):444-59.
43. Turk DC, Swanson KS, Gatchel RJ. Predicting opioid misuse by chronic pain patients: a systematic review and literature synthesis. Clin J Pain. 2008;24(6):497-508.

44. Jamison RN, Kauffman J, Katz NP. Characteristics of methadone maintenance patients with chronic pain. J Pain Symptom Manag. 2000;19(1):53-62.

45. Rosenblum A, Joseph H, Fong C, Kipnis S, Cleland C, Portenoy RK. Prevalence and characteristics of chronic pain among chemically dependent patients in methadone maintenance and residential treatment facilities. Jama. 2003;289(18):2370-8.

46. Butler SF, Budman SH, Fernandez KC, Houle B, Benoit C, Katz N, Jamison RN. Development and validation of the Current Opioid Misuse Measure. PAIN. 2007;130(1):144-156. https://doi.org/10. 1016/j.pain.2007.01.014.

47. Back SE, Gros DF, Price M, LaRowe S, Flanagan J, Brady KT, et al. Laboratory-induced stress and craving among individuals with prescription opioid dependence. Drug Alcohol Depend. 2015;155:60-7.

48. Hyman SM, Fox H, Hong K-IA, Doebrick C, Sinha R. Stress and drug-cue-induced craving in opioid-dependent individuals in naltrexone treatment. Exp Clin Psychopharmacol. 2007;15(2):134-43.

49. Tiffany ST, Wray JM. The clinical significance of drug craving. Ann N Y Acad Sci. 2012;1248:1-17.

50. Bradford DE, Shapiro BL, Curtin JJ. How bad could it be? Alcohol dampens stress responses to threat of uncertain intensity. Psychol Sci. 2013;24(12):2541-9.

51. Mantsch JR, Baker DA, Funk D, Lê AD, Shaham Y. Stress-induced reinstatement of drug seeking: 20 years of progress. Neuropsychopharmacology. 2016;41(1):335-56. https://doi.org/ 10.1038/npp.2015.142.

52. Ebner-Priemer UW, Eid M, Kleindienst N, Stabenow S, Trull TJ. Analytic strategies for understanding affective (in) stability and other dynamic processes in psychopathology. J Abnorm Psychol. 2009;118(1):195-202.

53. Lamers F, Swendsen J, Cui L, Husky M, Johns J, Zipunnikov V, et al. Mood reactivity and affective dynamics in mood and anxiety disorders. J Abnorm Psychol. 2018;127(7):659-69.

54. Ebner-Priemer UW, Sawitzki G. Ambulatory assessment of affective instability in borderline personality disorder: the effect of the sampling frequency. Eur J Psychol Assess. 2007;23(4):238-47. https://doi.org/10.1027/1015-5759.23.4.238.

55. Intille, S., Haynes, C., Maniar, D., Ponnada, A., \& Manjourides, J. (2016). $\mu$ EMA: microinteraction-based ecological momentary assessment (EMA) using a smartwatch. Proc ACM Int Conf Ubiquitous Computing, 1124-1128.

56. Epstein DH, Tyburski M, Kowalczyk WJ, Burgess-Hull AJ, Phillips KA, Curtis BL, Preston KL. Prediction of stress and drug craving ninety minutes in the future with passively collected GPS data. NPJ Digit Med. 20203,26. https://doi.org/10.1038/s41746020-0234-6

57. Suffoletto B, Dasgupta P, Uymatiao R, Huber J, Flickinger K, Sejdic E. A preliminary study using smartphone accelerometers to sense gait impairments due to alcohol intoxication. J Stud Alcohol Drugs. 2020;81(4):505-10. https://doi.org/10.15288/jsad.2020.81.505.

58. Zulueta J, Piscitello A, Rasic M, Easter R, Babu P, Langenecker $\mathrm{SA}$, et al. Predicting mood disturbance severity with mobile phone keystroke metadata: a biaffect digital phenotyping study. J Med Internet Res. 2018;20(7):e241.

59. Davis-Stober CP, Regenwetter M. The "paradox" of converging evidence. Psychol Rev. 2019;126(6):865-79. https://doi.org/10. 1037/rev0000156.

Publisher's Note Springer Nature remains neutral with regard to jurisdictional claims in published maps and institutional affiliations. 